\title{
Article
}

\section{Distraction in Verbal Short-Term Memory: Insights from Developmental Differences}

\author{
Elliott, Emily, Hughes, Robert W., Briganti, A, Joseph, Tanya \\ Nicolette, Marsh, John Everett and Macken, William J. \\ Available at http://clok.uclan.ac.uk/13937/ \\ Elliott, Emily, Hughes, Robert W., Briganti, A, Joseph, Tanya Nicolette, Marsh, \\ John Everett ORCID: 0000-0002-9494-1287 and Macken, William J. (2016) \\ Distraction in Verbal Short-Term Memory: Insights from Developmental \\ Differences. Journal of Memory and Language, 88 . pp. 39-50. ISSN 0749-596X
}

It is advisable to refer to the publisher's version if you intend to cite from the work. http://dx.doi.org/10.1016/j.jml.2015.12.008

For more information about UCLan's research in this area go to

http://www.uclan.ac.uk/researchgroups/ and search for < name of research Group $>$.

For information about Research generally at UCLan please go to http://www.uclan.ac.uk/research/

All outputs in CLoK are protected by Intellectual Property Rights law, including Copyright law. Copyright, IPR and Moral Rights for the works on this site are retained by the individual authors and/or other copyright owners. Terms and conditions for use of this material are defined in the policies page. 
Distraction in Verbal Short-Term Memory: Insights from Developmental Differences

Emily M. Elliott ${ }^{1}$, Robert W. Hughes ${ }^{2}$, Alicia Briganti ${ }^{1}$, Tanya Joseph ${ }^{3}$, John E. Marsh ${ }^{3}$, and Bill Macken ${ }^{4}$

1. Louisiana State University; 2. Royal Holloway, University of London; 3. University of Central Lancashire; 4. Cardiff University

Author Note

Portions of this work were presented at the 52 and $53^{\text {rd }}$ Annual Psychonomic Society Conference, Seattle, WA, and Minneapolis, MN, respectively. Emily M. Elliott and Alicia Briganti, Department of Psychology, Louisiana State University, USA. Alicia Briganti is now at the Department of Social Sciences, Dalton State College, USA. Robert W. Hughes, Department of Psychology, Royal Holloway, University of London, UK. Tanya N. Joseph and John E. Marsh, School of Psychology, University of Central Lancashire, UK. Bill Macken, School of Psychology, Cardiff University, UK. Correspondence concerning this article should be addressed to Emily M. Elliott, Department of Psychology, 236 Audubon Hall, Louisiana State University, Baton Rouge, LA, 70803, USA. Email: eelliott@1su.edu. Phone: 1-225-578-7460. 


\begin{abstract}
The contribution of two mechanisms of auditory distraction in verbal serial short-term memory-interference with the serial rehearsal processes used to support short-term recall and general attentional diversion-was investigated by exploiting differences in auditory distraction in children and adults. Experiment 1 showed that serial rehearsal plays a role in children's as well as adults' distractibility: Auditory distraction from irrelevant speech was greater for both children and adults as the burden on rehearsal increased. This pattern was particularly pronounced in children, suggesting that underdeveloped rehearsal skill in this population may increase their distractibility. Experiment 2 showed that both groups were more susceptible to changing- than steady-state speech when the task involved serial rehearsal-indicating that both groups suffer interference-by-process - but that children, but not adults, were also susceptible to any sort of sound (steady or changing) in a task thought to be devoid of serial rehearsal. The overall pattern of results suggests that children's increased susceptibility to auditory distraction during verbal short-term memory performance is due to a greater susceptibility to attentional diversion; in this view, under-developed rehearsal-skill increases children's distractibility by exacerbating their under-developed attentional control rather than by increasing interference-byprocess.
\end{abstract}

Keywords: irrelevant sound effect, verbal short-term memory, auditory distraction, developmental differences, attentional control, rehearsal 
Distraction in Verbal Short-Term Memory: Insights from Developmental Differences

The ability to maintain verbal information in order over a short time period is an important cognitive skill, underpinning many fundamental higher-order functions including language comprehension, language learning, and verbally-mediated problem-solving (e.g., Baddeley, 2007). Common to numerous theoretical accounts is the notion that a key process in verbal serial short-term memory is rehearsal, in which the vocal system is used either as a means of offsetting the decay of phonological representations in a labile short-term store (e.g., Baddeley, 2003, 2007; Camos, Lagner, \& Barrouillet, 2009; but see Lewandowsky \& Oberauer, 2015; Oberauer \& Lewandowsky, 2012) or, according to other theories, of constructively binding a grammatically and semantically unconstrained sequence into a coherent motor-plan for action (e.g., Hughes, Marsh, \& Jones, 2009; Jones, Hughes, \& Macken, 2006). However, there is evidence that such active rehearsal processes are peculiarly vulnerable to disruption by passively processed irrelevant auditory stimuli, whether speech or non-speech (e.g., tones), even when the memory task is presented visually (e.g., Beaman \& Jones, 1997; Colle \& Welsh, 1976; Jones \& Macken, 1993; Jones, Macken, \& Nicholls, 2004; Salamé \& Baddeley, 1982). The study of this irrelevant sound effect (ISE), particularly across development, is instructive for theories of shortterm memory and selective attention as it provides a vehicle for understanding the ability to maintain and reproduce aspects of one's environment in the service of complex cognition, the role of attentional control in such maintenance, and how such processes emerge over time. The current research examines the ISE in both children and adults with the aim of establishing the extent to which verbal short-term memory is susceptible to distraction due to the vulnerability of 
serial rehearsal and/or to poor general attentional control. Additionally, we examine whether these two factors play different roles at different points of cognitive development.

There is evidence that young children may be especially affected by irrelevant sound as compared to college students and older adults, who do not differ in their susceptibility to such distraction (Beaman, 2005; Elliott, 2002; Elliott, Bhagat, \& Lynn, 2007; Elliott \& Briganti, 2012; Rouleau \& Belleville, 1996; cf. Klatte, Lachmann, Schlittmeier, \& Hellbruck, 2010). In the typical irrelevant sound experiment, participants are presented visually with a series of around 5 to 9 verbal items (digits or words) at the rate of about 1 item per second. Following the last item, or following a short retention interval, the items must be recalled in serial order. The ISE refers to the robust finding that the presentation of sound that is not necessarily loud [e.g., 55-65 $\mathrm{dB}(\mathrm{A})]$, is irrelevant to the task, and that participants are therefore told to ignore, disrupts serial recall appreciably (e.g., Beaman \& Jones, 1997; Colle \& Welsh, 1976; Jones, Madden, \& Miles, 1992; Salamé \& Baddeley, 1982). A prominent account of the ISE in adults posits that it is due to a conflict of two concurrent processes of seriation (e.g., Hughes, 2014; Hughes \& Jones, 2001). A key line of evidence for this interference-by-process account is the changing-state effect: While some degree of disruption from 'steady-state' sound (e.g., “ $K K K K K \ldots$..”) compared to quiet has sometimes been observed (e.g., Hughes, Tremblay, \& Jones, 2005; LeCompte, 1994), a plethora of studies has shown that changing-state sound (e.g., " $K P R L$ $N .$. ") — that forms a series of auditory stimuli — is particularly disruptive of serial recall (e.g., Elliott, 2002; Hughes et al., 2005; Jones et al , 1992; Jones \& Macken, 1993; LeCompte, 1994). In addition, a demand for seriation in the focal task — in the form of serial rehearsal—plays a role in the disruption: On the interference-by-process account, engagement in serial rehearsal is vulnerable to interference from the pre-attentively seriated auditory information (Beaman \& 
Jones, 1997). Similarly, in tasks that are typically thought to be supported by serial rehearsal, impeding the capacity for rehearsal through articulatory suppression diminishes or eliminates the disruption from changing-state sound (Jones et al., 2004).

Given the important role ascribed to serial rehearsal in the ISE in adults, one of the chief questions we address in the present study is whether the inchoate state of children's rehearsal skill plays a role in their increased susceptibility to the effect (Tam, Jarrold, Baddeley, \& Sabatos-DeVito, 2010). The efficiency of rehearsal processes is thought to increase with development during childhood (e.g., Flavell, Beach, \& Chinsky, 1966; Tam et al., 2010; but see also Jarrold \& Citroën, 2013). Recent evidence suggests that relatively young children's (age 8) rehearsal processes are less practiced and continue to change from a single-item repetition style of rehearsal to a more cumulative style as they approach later childhood (e.g., age 10; Lehmann \& Hasselhorn, 2012). Furthermore, there is evidence that younger children use greater levels of processing resources when rehearsing, relative to older children. For example, Guttentag (1984) used a dual-task approach to quantify the mental cost incurred by rehearsal in three groups of children, ranging in age from approximately 7 to 12 years. Using a free recall task, he found that a cumulative rehearsal strategy demanded greater mental effort in younger children as evidenced by a greater cost of a secondary finger-tapping task.

In adults, it is argued that the more skilled or fluent the rehearsal process-based either on the nature of the to-be-remembered material or individual differences in speech-planning and production - the less vulnerable they will be to the ISE. That is, disruption by the seriation cues yielded by changing-state sound is assumed to be a negative function of the extent to which the to-be-remembered items can be fluently co-articulated during serial rehearsal (e.g., Jones, Beaman, \& Macken, 1996). It follows, therefore, that it is the less efficient rehearsal abilities of 
children that renders them more vulnerable to disruption by changing-state sound. Another possibility, however, is that if children gain less from using rehearsal—due to its underdevelopment - they stand to lose less in the presence of a factor assumed to further reduce the efficiency of that rehearsal process. However, at least within the interference-by-process framework, this would predict a smaller ISE in children (that are beginning to rehearse) compared to adults which, to our knowledge, has never been observed.

Our reasoning so far has been that underdeveloped rehearsal skill could leave children compared to adults particularly susceptible to interference-by-process (e.g., Jones \& Tremblay, 2000): The less fluent the transition from one to-be-remembered item to the next during serial rehearsal the more opportunity there would be for seriation cues yielded obligatorily by changing-state sound to intrude into that rehearsal process and thereby impair recall performance (see, e.g., Jones et al., 1996; Macken et al., 1999). An alternative possibility, however, is that the underdeveloped nature of rehearsal in children could impose a general attentional or executiveprocessing load. To elaborate, in addition to the quantitative and qualitative changes in rehearsal, evidence suggests that children have a smaller working memory capacity (Cowan et al., 2005) and hence a more limited ability to filter out irrelevant stimuli, especially when placed under conditions of high focal-task demand (Cowan, Morey, AuBuchon, Zwilling, \& Gilchrist, 2010). Thus, children's attentional abilities can be equivalent to adults in some circumstances but under difficult conditions developmental differences are likely to emerge.

The fact that there is evidence that both attentional control and rehearsal skill are underdeveloped in children compared to adults suggests that an emerging duplex-mechanism account of auditory distraction could provide a fruitful framework for understanding developmental differences in distractibility (see also Meinhardt-Injac et al., 2015). The duplex-mechanism 
account (e.g., Hughes, Hurlstone, Marsh, Vachon, \& Jones, 2013; Hughes, Vachon, \& Jones, $2005,2007)$ posits that the cognitive system is susceptible to two distinct mechanisms of auditory distraction: interference with the particular process (e.g., rehearsal) deployed to perform the focal task (interference-by-process) and the diversion of attention away from a focal task such as typically found with an unexpected deviant sound (e.g., Näätänen, 1990) or meaningfully salient sound (e.g., one's own name; Moray, 1959; Röer, Bell, \& Buchner, 2013). For example, one line of evidence that dissociates these two forms of distraction and which is particularly germane in the present context is that adults with low working memory capacity-as measured by complex working memory tasks such as operation span (OSPAN; Turner \& Engle, 1989)— show a greater vulnerability to distraction via attentional diversion (e.g., by a deviant sound) but not to one of the key signatures of interference-by-process, namely, distraction by changingcompared to steady-state sound (Hughes et al., 2013; Sörqvist, 2010; Sörqvist, Marsh, \& Nöstl, 2013). Given children's low working memory capacity compared to adults (Cowan et al., 2005), it seems reasonable to conjecture that children are also likely to be particularly prone to attentional diversion, especially under a high rehearsal (or any other source of cognitive) load which would be expected to draw upon the capacity for staying focused on task-goals (cf. Lavie, 2010; Lavie, Hirst, de Fockert, \& Viding, 2004). Furthermore, relatively early in cognitive development, salient events such as unexpected deviants or hearing one's own name may not be necessary to divert attention; any extraneous stimulation may constitute a potent source of distraction (Wetzel \& Schröger, 2007).

In the present study, therefore, we examine the role of both rehearsal processes and attentional control in the developmental difference seen between young children and adults in relation to auditory distraction in serial short-term memory. The children in the current 
experiments were between 7 and 8 years of age and hence were within the timeframe of developmental changes in rehearsal (e.g., Lehmann \& Hasselhorn, 2012). To anticipate, we will show that while underdeveloped rehearsal appears to indeed play a role in children's increased distractibility compared to adults, this is not through its exacerbation of an interference-byprocess mechanism, but rather its exacerbation of their proneness to attentional diversion.

\section{Experiment 1}

In Experiment 1, we manipulated the point in a serial recall task at which the irrelevant sounds were presented. In line with the interference-by-process account, the magnitude of the disruption in adults has been shown to be a positive function of the extent to which the sound coincides with points at which the demand on serial rehearsal is relatively great (Macken, Mosdell, \& Jones, 1999). Specifically, in Macken et al. (1999), the effects of irrelevant speech were investigated at different temporal segments within a 25 -second trial. In their design, each trial consisted of a 5-second pre-presentation segment, a 10-second presentation phase (divided into two 5-second segments) — that is, the phase during which the to-be-remembered items were presented - and a 10-second retention interval (also divided into two 5-second segments). The rationale was that rehearsal load would be expected to increase with each segment as more and more items were presented and subsequently needed to be retained until the recall cue. During one of these 5-second segments per trial, changing-state irrelevant speech in the form of a narrative was presented. The results showed that irrelevant speech presented in the second segment of the presentation phase or either segment of the retention interval caused significantly more disruption (compared to quiet) than speech played during the pre-presentation segment or the first presentation segment. 
Here we use the manipulation of the timing of sound-presentation to examine whether the development of serial rehearsal plays a role in children's increased susceptibility to auditory distraction. If it does, the difference between children and adults' performance should be particularly apparent during phases of the task that impose a relatively high rehearsal load. The present experiment contains two minor changes to the design of Macken et al. (1999). First, a succession of individual words replaced the narrative speech. Second, given the necessity of having a shorter to-be-remembered list for the children (4 items) compared to adults (8 items), the trials were $20 \mathrm{~s}$ long (five $4 \mathrm{~s}$ segments) for the adults, and $12 \mathrm{~s}$ long for the children (see Method for details). This design provided a matched number of irrelevant sounds presented simultaneously with each to-be-remembered item across the two age-groups.

\section{Method}

Participants. The 25 child participants (16 female) were approximately 7 years old ( $M$ age $=7.4$ years ) and recruited from two East Baton Rouge Parish academic magnet elementary schools. These academic magnet elementary schools require that students pass a screening test for admission, and that they maintain at least a 2.5 GPA. In this way, these elementary school students represent a more selective group than the general population of elementary school students. In addition, 137 Louisiana State University undergraduates participated and 37 were included in the current data analyses on the basis that these represented the upper quartile of performance in the silent condition, as a means of matching performance levels to the children's performance in the silent condition (see Results section for more details). Thus, there was a total of 62 participants across the two age-groups.

Design and Materials. Children were first given a digit span assessment to determine their individual level of performance. For the digit span task, visually-presented lists of the digits 
1-9 were presented in random order, beginning with list length three, and four lists of each length were presented. Participants were asked to vocalize their responses in the order that the numbers appeared during list presentation. List presentation ended when a participant recalled more than two lists incorrectly. Digit span was defined as the highest list length with two or more lists correctly recalled.

Children were then asked to remember lists of 4 digits in the irrelevant sound task. Eprime 2.0 software was used for presentation of the visual and auditory stimuli (Schneider, Eschman, \& Zucolotto, 2002). Samples of irrelevant sound were produced by random selection from the word set bomb, corn, fish, and tape. The irrelevant sounds were digitally recorded in a female voice at $22 \mathrm{KHz}$ using a sound-editing program (Cool-Edit). The level of sound was measured at approximately 65dB (A) with a Quest sound level meter and an earphone coupler.

For the children, each irrelevant sound trial consisted of a 4 s pre-presentation phase, a 4digit list presentation phase, and a $4 \mathrm{~s}$ retention interval. Thus, each trial lasted $12 \mathrm{~s}$ before recall was required. The $12 \mathrm{~s}$ of each trial were divided into five segments. The $4 \mathrm{~s}$ pause before presentation was termed Pre-presentation ${ }^{1}$. The first $2 \mathrm{~s}$ of the digit presentation phase was termed Presentation 1, with the final $2 \mathrm{~s}$ of the presentation phase being Presentation 2. The $4 \mathrm{~s}$ retention interval was similarly divided to establish Retention 1 and Retention 2 . There were five sound conditions with words presented during one randomly selected $2 \mathrm{~s}$ segment during each trial, with the exception of the 4 s Pre-presentation interval, which contained sounds throughout the $4 \mathrm{~s}$. The words were edited to span $400 \mathrm{~ms}$ with a $100 \mathrm{~ms}$ silent period between sound tokens. This resulted in two sound tokens presented with each TBR digit in the presentation

\footnotetext{
${ }^{1}$ At first glance, it may seem odd to include a pre-presentation condition because, by definition, in this condition the sound is not concurrent with any to-be-remembered material (or its rehearsal). However, its inclusion represents a legacy of Macken et al.'s (1999) design which tested a temporal distinctiveness account of the ISE (LeCompte, 1996) which predicted (incorrectly) that disruption from sound presented during a prepresentation phase would be as large as when the sound is presented just after presentation.
} 
phase. The sounds were selected quasi-randomly with the exception that the same word could not be presented in immediate succession (e.g., sounds 4 and 5 were not the same word). There was also a quiet control condition in which no sounds were presented. Thus, there were six experimental conditions in all.

All participants were presented with digit lists one digit at a time in the center of a computer screen at the rate of 1 digit/s. Participants experienced six lists in each of the six experimental conditions. The lists were presented in quasi-random blocks such that participants received a digit list during each of the auditory conditions before proceeding to the next block of lists.

The design was the same for the adults with the exception that the list length was 8 items. The number of sounds (two) per TBR item was the same as for the children and hence, given the greater list-length, the sound segments were longer for the adults (4 s each; five segments for a total of $20 \mathrm{~s}$ per trial). The adults received 10 trials of each type, presented quasi-randomly within a block of trials.

Procedure. Child participants were tested individually and the Experimenter was present at all times. Prior to beginning the ISE task, an assessment of digit span was completed for the children only. Then, participants began the ISE task. Instructions were presented on the computer screen and any questions were answered before proceeding. Throughout the session, participants wore headphones connected to a laptop computer. Participants experienced three practice trials in the quiet control condition for familiarization with the procedure before proceeding to the test trials.

Participants initiated each trial by pressing the space bar. After the space bar was pressed, the screen remained blank for 4 seconds before list presentation began. After list 
presentation was complete, the word wait appeared on the screen for 4 seconds (retention interval) followed by the word recall. Participants were instructed to use the number keypad on the computer keyboard to enter the digits of each list in the order they appeared. If they could not remember a digit in a list, they were instructed to guess. Halfway through, participants were offered the opportunity to take a break. Participants were instructed to ignore all sounds and were assured that they would not be tested on them. The entire session (including the digit span task) lasted approximately $40 \mathrm{~min}$. The procedure was similar for the adults; however, they did not complete a digit span assessment and their experimental session lasted approximately $1 \mathrm{hr} 15$ min. Adults were run in groups ranging from 1-6 participants, and a trained experimenter was present during the entire session.

\section{Results}

Supporting previous research in adults (e.g., Ellermeier \& Zimmer, 1997), no gender differences were observed in the size of the ISE in the children in this sample and thus the data were collapsed across the factor of gender in the following analyses. The results of the digit span assessment in the children indicated that a list length of 4 items was appropriate $(M=4.32$, S.D. $=.85$ ). The majority of the children had a span length of either 4 or 5 items (the frequency counts were four children with a span of 3 , eleven with a span of 4 , eight with a span of 5 , and two with a span of 6).

From the sample of 137 college students, the mean performance in the silent condition was used to determine a comparable baseline to the $2^{\text {nd }}$ graders. The upper quartile was retained, with $\mathrm{N}=37(M=.89, S D=.05)$. For the $2^{\text {nd }}$ grade group $(\mathrm{N}=25)$, comparable performance in silence was observed $(M=.87, S D=.12)$. This similar-baseline-matching approach was also employed in Elliott and Briganti (2012). 
Figure 1 shows the recall performance (collapsed across serial position) of the children and adults in the silence-throughout condition and across the five sound-segment conditions. It is clear that the detrimental effect of the irrelevant speech was larger late into the presentation phase and during the retention interval than before or during the early-presentation phase, and that this pattern is particularly pronounced for children. Statistical analyses confirmed this impression: A 2 x 6 mixed ANOVA revealed main effects of auditory condition, $F(5,300)=$ $30.69, M S E=.01, \eta_{\mathrm{p}}^{2}=.34, p<.01$, and age-group, $F(1,60)=4.18, M S E=.07, \eta_{\mathrm{p}}{ }^{2}=.07, p=$ .04 , as well as an interaction of these two factors, $F(5,300)=2.34, M S E=.01, \eta_{\mathrm{p}}^{2}=.04, p=.04$, in line with the greater disruption suffered by children at Presentation 2, Retention 1 and Retention 2.

Additionally, the arcsine transformation was applied to the data, to correct for any possible violations of the assumptions of ANOVA, or problems with interpretation that can arise when applying an ANOVA approach to proportion correct data (Jarrold \& Citroën, 2013). The results were the same as for the 2 × 6 ANOVA described above.

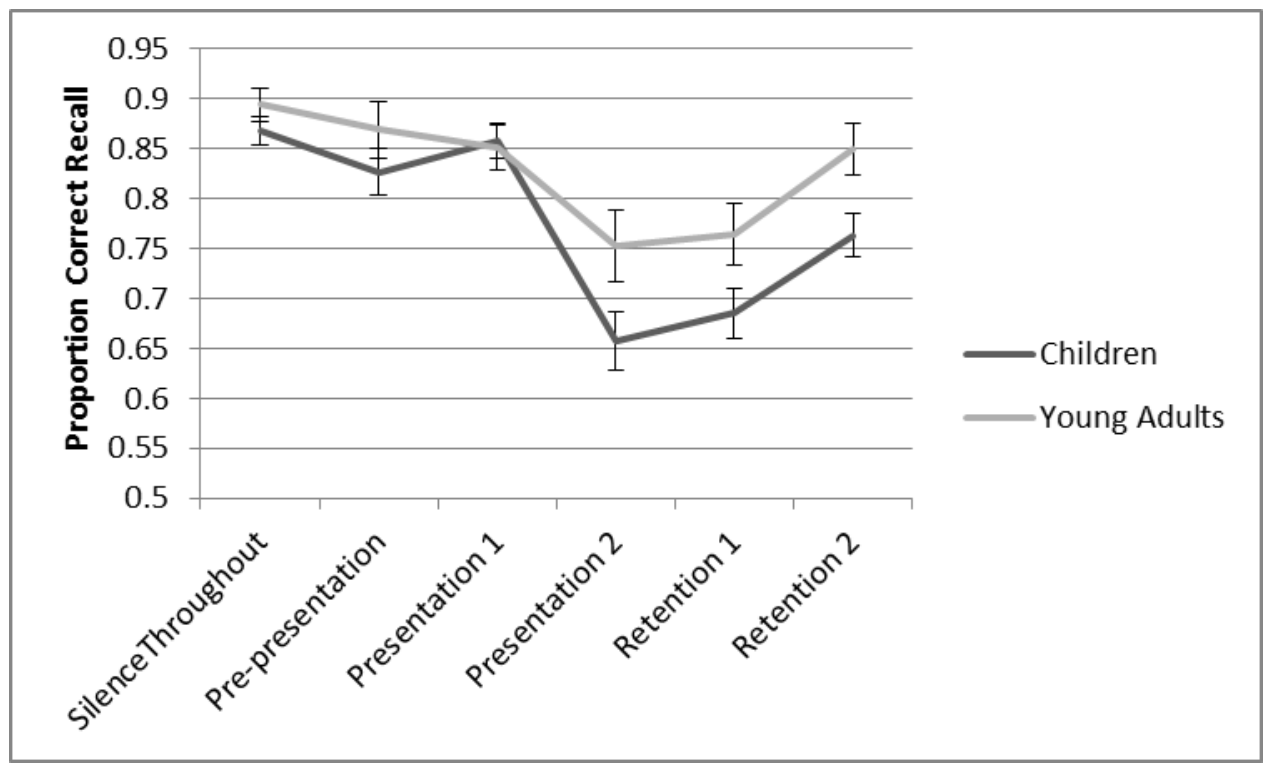


Figure 1. Mean proportion correct serial recall performance for the children and adults in the silence-throughout condition and across the five sound-segment conditions. Error bars represent standard error of the mean.

The data from each age-group were then analyzed in turn. For the children, the repeatedmeasures ANOVA comparing the different sound-segments indicated a significant main effect, $F(5,120)=13.24, M S E=.02, \eta_{\mathrm{p}}^{2}=.36, p<.001$, and the follow-up comparisons with the Bonferroni correction indicated that performance in Pre-presentation and Presentation 1 did not differ from that in the silence-throughout condition, but that Presentation $2(p<.001)$, Retention $1(p=.001)$, and Retention $2(p<.05)$ were indeed significantly different from silence. There were no significant differences between Presentation 2, Retention 1, and Retention 2.

The equivalent analysis for the adult group also revealed a significant effect of sound segment, $F(5,180)=17.61, M S E=.007, \eta_{\mathrm{p}}{ }^{2}=.33, p<.001$. Significant disruption from irrelevant sound (compared to silence) occurred during Presentation $2(p<.001)$ and Retention 1 $(p<.001)$. Adults demonstrated no disruption at Pre-presentation or Presentation 1 (relative to silence) and irrelevant sound also had no effect on the adults' performance when presented late into the retention interval (i.e., comparison of Retention 2 and silence, all $p>.05$ ).

\section{Discussion}

First, the results of Experiment 1 confirm previous observations that children are more susceptible to the disruptive effect of irrelevant sound than adults (Elliott, 2002; Elliott et al., 2007; Elliott \& Briganti, 2012) contrary to a single report by Klatte et al. (2010) suggesting no group differences (see also General Discussion). Second, the experiment is the first to show that children as well as adults (cf. Macken et al., 1999) are more negatively affected by irrelevant sound at the end of the presentation interval and during the retention interval, at which points the demand on serial rehearsal processes is relatively great. Furthermore, it is only during these high 
rehearsal-load phases that any difference in the ISE between children and adults is seen. The lack of a difference between children and adults in the early period of the trial (Pre-presentation or Presentation 1) appears to reflect a type of a ceiling effect, as neither group shows a detriment in performance relative to silence. The results therefore are in line with the view that the ISE is underpinned by the use of serial rehearsal but also that the greater vulnerability of children to the ISE may be related to the underdevelopment of rehearsal skill at this developmental stage (e.g., Tam et al., 2010).

One difference that emerged in the pattern of disruption across the auditory conditions was that adults' performance at Retention 2 was no different from the silent condition, whereas the children's was different. Macken et al.'s (1999) study with adults also found that performance was better in (the equivalent of) their Retention 2 condition than their Retention 1 (and Presentation 2) condition. They suggested that the rehearsal cohort may become increasingly stable across a retention interval and hence become less vulnerable to disruption. Speculatively, the group difference found in the present experiment with regard to Retention 2 may also be couched in terms of the underdeveloped-rehearsal view: The rehearsing of a given sequence may not become automatized as rapidly for children as for adults, such that children remain susceptible to irrelevant sound even after a relatively extended period of rehearsing the same sequence. However, it should be noted that the pattern for children from Presentation 2 to Retention 2 is still very similar to that for adults - as clearly evident in Figure 1 - suggesting that there may indeed be some stabilization of the rehearsal cohort in children, albeit not enough to reach the same level of performance as in silence. Second, in Macken et al. (1999), performance at Retention 2 did not reach the same level as silence, an observation that aligns more with performance in our child group than our adult group. Thus, while this particular aspect of our 
data is broadly consistent with the underdeveloped-rehearsal view, this 'stabilizing-cohort' interpretation should be treated with some caution.

One interpretation of the results of Experiment 1 is that underdeveloped rehearsal-skill in children makes them more vulnerable to interference-by-process: order cues derived involuntarily from the sound is particularly liable to encroach into their relatively fragile rehearsal process (e.g., Jones \& Tremblay, 2000). However, another interpretation-motivated by the duplex-mechanism account (e.g., Hughes et al., 2007, 2013)—is that, given that same underdevelopment of rehearsal-skill, increasing the rehearsal load makes it particularly difficult for children compared to adults to maintain attention on the focal task (e.g., Engle, 2002; Guttentag, 1984; Lavie, 2010; Lavie et al., 2004). In this view, the effect of underdeveloped rehearsal is indirect: it is one of possibly many sources of increased attentional or cognitive load that would make them more vulnerable to distraction. We examine this interpretation further in Experiment 2.

\section{Experiment 2}

In Experiment 2, we examine the extent to which the particular vulnerability of children's serial short-term memory to auditory distraction is due at least in part to a greater likelihood of attentional diversion as opposed to greater interference-by-process. Our approach was to examine auditory distraction effects in children and adults in the context of two short-term memory tasks that are very closely matched except for the involvement of serial rehearsal. As noted in the Introduction, one pillar of support for interference-by-process is that tasks making little or no demand on serial order processing — and hence serial rehearsal—are affected little, if at all, by changing-state irrelevant sound. However, whereas this prediction has been confirmed several times in adults (e.g., Beaman \& Jones, 1997; Hughes et al., 2007; Jones \& Macken, 
1993), it has not been tested in children, until now. If young children's susceptibility to auditory distraction is due at least in part to poorer general attentional control—rather than greater interference-by-process - then children, but not adults, should exhibit marked distraction even when the focal task is devoid of serial rehearsal.

The two short-term memory tasks contrasted here were the probed-order recall task (e.g., Murdock, 1968) and the missing-item task (e.g., Buschke, 1963). In the probed-order task, participants are presented with a series of items (e.g., '587130649'; note that the list-length was reduced for the children - see Method section) and then, at test, probed with one of the items (e.g., '6') to which they must respond with the item that followed it in the list (i.e., '4'). This task, like serial recall (cf. Experiment 1), requires the maintenance of the serial order of the items and, accordingly, there is ample evidence that this task, like serial recall, is supported by a serial rehearsal strategy (e.g., Hughes, Marsh, \& Jones, 2011; Macken \& Jones, 1995). In the missingitem task, participants are asked instead to report the one item (from the 0-9 set) that was missing (e.g., '2' is missing from '587130649'). Thus, this task is identical to the probed-order recall task in terms of list-presentation and the requirement for a single response but, critically, does not require the retention of item-order and does not therefore tend to invoke a serial rehearsal strategy. Instead, accounts of missing-item recall tend to invoke processes related to individual short-term item familiarity (or, more accurately, unfamiliarity) — which may be supported by a 'ticking off' of each incoming item against a representation of the fixed ordinal sequence (i.e., '1, 2, 3...'; see, e.g., Buschke \& Hinrichs, 1968; Humphreys \& Schwartz, 1971)—rather than to processes relating to order within the presented sequence. Independent corroboration for this distinction between the tasks in terms of the role of serial rehearsal comes from the fact that probed-order recall is affected far more by articulatory suppression than is the missing-item task 
(Beaman \& Jones, 1997; Hughes et al., 2007; Jones \& Macken, 1993; Klapp, Marshburn, \& Lester, 1983; Macken \& Jones, 1995). Of particular relevance for present purposes, studies with adults have shown, in line with the interference-by-process account, that probed-order recall is also far more vulnerable to disruption by irrelevant speech than the missing-item task (Beaman \& Jones, 1997): The two sources of order information from the two modalities come into conflict in the probed-order recall task whereas the absence of serial rehearsal in the missing-item task immunizes it from any such conflict. However, if children are also particularly susceptible to attentional diversion then they may be expected to show marked disruption by sound in the missing-item task as well as the probed-order recall task. We also included a steady-state as well as a changing-state sound condition in this experiment. If children are particularly susceptible to distraction due to poor general attentional control, they may be expected to show greater disruptibility by sound generally (regardless of its particular structure).

\section{Method}

Participants. A total of 128 participants, 64 college students from Louisiana State University (9 males; $M=19.69$ years), and 64 children were recruited for this study. There were 35 children (13 males; $M=7.7$ years) from East Baton Rouge Parish academic magnet elementary schools in the US, and 29 children from Preston, Lancashire, UK (13 males; $M=7.5$ years). None had participated in Experiment 1.

Design and Materials. The participants completed two visually-presented short-term memory tasks - the missing-item and the probed-order recall task —in the presence of three auditory conditions: silence, steady-state sound, and changing-state sound. For each age-group, the experimental design included task and auditory condition as within-participant variables. 
All tasks were presented on computer via Eprime (Schneider et al., 2002). The to-beremembered visual stimuli were digits from the set 0-9 in the adult group, while the children were shown digits from the set $0-5$. The auditory stimuli comprised digitized recordings of a female voice, and were presented via headphones. The changing-state stimuli consisted of the letters $A, B, C, G, J, K, L, M, Q$, and $S$, and the steady-state stimuli were repetitions of either the letter $Q$ or the letter $M$. Auditory and visual stimuli were presented at the rate of $1 / \mathrm{s}$, with simultaneous onsets of the two types of stimuli in the sound conditions. The level of sound was measured at approximately $72 \mathrm{~dB}(\mathrm{~A})$ with a Quest sound level meter and an earphone coupler.

Procedure. In the probed-order recall task, adult participants were presented with a series of nine digits from the ten-item set 0-9 (e.g., '587130649') and were then asked "which item followed $x$ ?" where $x$ denotes one of the list-items chosen at random on each trial. In the missing-item task, adults were asked to report the one digit that was missing (e.g., '2' is missing from '587130649'; Buschke, 1963; Hughes et al, 2011; Klapp et al., 1983). The procedure was the same for the children, with the exception of the list-length (five items were presented from the six-item set 0-5 for both tasks). The adults and the UK child-sample typed in their responses on the keyboard, while the Experimenter typed in the US children's spoken responses ${ }^{2}$.

As in Experiment 1, the children completed a measure of digit span prior to completing the irrelevant sound tasks. Whether participants began with the missing-item or probed-order recall task was counterbalanced across participants, and the two tasks were alternated in blocks of 6 trials each, throughout the experimental session. However, first a set of three practice trials

\footnotetext{
2 In the UK sample, the children were run in small groups and entered their responses using the keypad. In the US sample, the children were run individually and the experimenter keyed in the response. Initial analyses were done to examine whether these minor procedural differences interacted with the children's performance across the auditory conditions, separately for each task. While the UK sample had lower levels of performance overall relative to the US sample, leading to significant main effects of child-sample in the two tasks, there were no significant interactions with auditory condition. Thus, child-sample was dropped as a factor for the analyses proper.
} 
was presented in silence for each task, to familiarize participants with the two tasks. This was followed by a practice session of 6 trials for each task in which the auditory stimuli were included but not scored. For the experiment proper, the auditory stimuli were quasi-randomly assigned, such that within each block of 6 trials there were two presentations of each auditory condition. Thus, the children completed 6 blocks of 6 trials each, for a total of 36 trials in each of the two tasks. The adults completed 10 alternating blocks of 6 trials each, for a total of 60 trials in each of the two tasks. For both groups of participants, the sessions were completed within 1 hr.

\section{Results}

The children's performance on the digit span task was evaluated first $(M=4.26, S D=$ $.97)$. The choice of presenting 5 items in the two memory tasks was an appropriate level of difficulty: The frequency counts indicated that the majority of the sample had a digit span of either 4 or 5 items (17 with a span of 3,20 with a span of 4, 20 with a span of 5 , and 7 with a span of 6).

Figures 2 and 3 show proportion correct recall as a function of age-group and auditory condition for the probed-order recall task and the missing-item task, respectively. A 2 (agegroup) x 2 (task) x 3 (auditory condition) mixed ANOVA revealed a main effect of age-group, $F(1,126)=6.21, M S E=.17, \eta_{p}{ }^{2}=.04, p=.01$, reflecting a higher mean level of performance in the adults $(M=.53)$ than in the children $(M=.46)$. The main effect of task was significant as well, $F(1,126)=50.20, M S E=.05, \eta_{p}{ }^{2}=.29, p<.01$, reflecting higher mean levels of performance in the missing-item task $(M=.55)$ than in the probed-order recall task $(M=.43)$. The main effect of auditory condition, $F(2,252)=33.59, M S E=.01, \eta_{p}{ }^{2}=.21, p<.01$, was investigated with pairwise comparisons using the Bonferroni correction. Recall was greatest in 
silence $(M=.54)$, differing significantly from that in the steady-state $(M=.47)$ and changingstate sound conditions $(M=.46$; both $p$ s $<.01)$ while the latter two conditions did not differ significantly from each other. However, these main effects were qualified by significant two-way interactions: First, age-group interacted with task, $F(1,126)=5.78, M S E=.05, \eta_{p}^{2}=.04, p<$ .02 , whereby the better recall performance of adults compared to children was more pronounced in the missing-item task. Second, age-group interacted with auditory condition, $F(2,126)=6.83$, $M S E=.02, \eta_{p}{ }^{2}=.05, p<.01$, replicating the general finding that children are more susceptible to auditory distraction than adults. Finally, task and auditory condition interacted as well, $F(2$, $252)=4.97, M S E=.01, \eta_{p}^{2}=.04, p<.01$, reflecting the greater susceptibility, in general, of probed-order recall to disruption by sound compared to the missing-item task (see also below). The three way interaction was not significant $(p=.26)$.

To investigate the two-way interactions further, analyses were then conducted separately for each task. A 2 × 3 mixed ANOVA on the data from the probed-order recall task (Figure 2) revealed a main effect of auditory condition, $F(2,252)=28.26, M S E=.01, \eta_{p}{ }^{2}=.18, p<.01$. Pairwise comparisons with the Bonferroni correction indicated that the three auditory conditions all differed significantly from one another $(M=.49, .43$, and .38 , respectively, for quiet, steadystate and changing-state sound, all $p s<.01)$. There was no main effect of age-group, $F(1,126)=$ $1.38, M S E=.08, \eta_{p}{ }^{2}=.01, p=.24$, and no interaction between auditory condition and agegroup, $F(2,252)=1.46, M S E=.01, \eta_{p}{ }^{2}=.01, p=.23$. The same pattern of results was observed when an arcsine transformation of the data was performed to address possible concerns related to the use of proportion correct data, as in Experiment 1 (Jarrold \& Citroën, 2013). The results were also the same when children who had a digit span of $3(n=17)$ were excluded. 


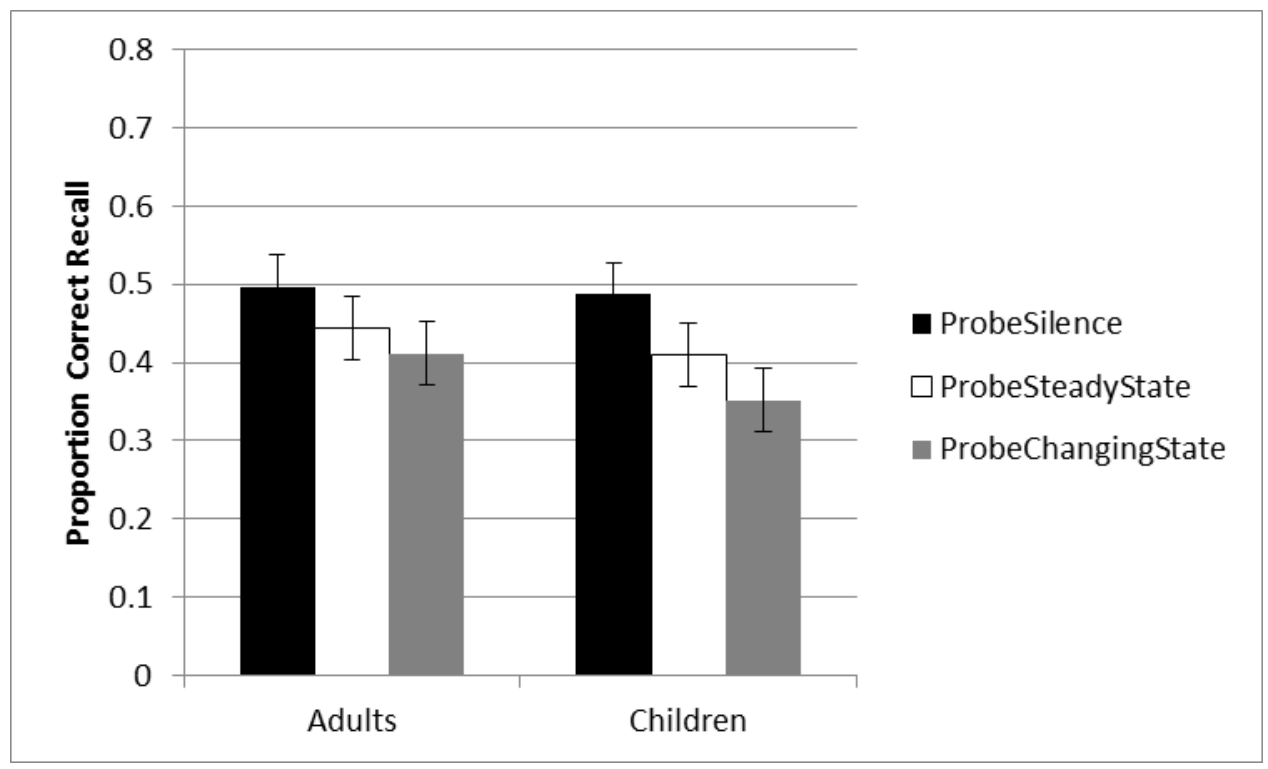

Figure 2. Recall performance in the probed-order recall task by auditory condition and age-group in Experiment 2. Error bars represent standard error of the mean.

For the missing-item task (Figure 3), a 2 x 3 mixed ANOVA showed a main effect of auditory condition, $F(2,252)=14.18, M S E=.01, \eta_{p}{ }^{2}=.10, p<.01$, as well as a main effect of age-group, $F(1,126)=8.89, M S E=.14, \eta_{p}{ }^{2}=.07, p<.01$, reflecting the higher performance of adults $(M=.61, S D=.23)$ compared to children $(M=.49, S D=.24)$. Additionally, there was a significant two-way interaction of auditory condition by age-group, $F(2,252)=7.27, M S E=$ $.012, \eta_{p}{ }^{2}=.06, p<.01$. A simple-effects analysis showed that the only significant difference between auditory conditions within the adult-group was that between steady-state and silence, $p$ $=.029$. While this particular effect was not expected, it is nevertheless clear from Figure 3 that the children showed greater disruption from sound than adults regardless of the nature of the sound, with performance in silence for children being greater than that in both the steady-state condition, $p<.0001$, and the changing-state condition, $p<.0001$. 


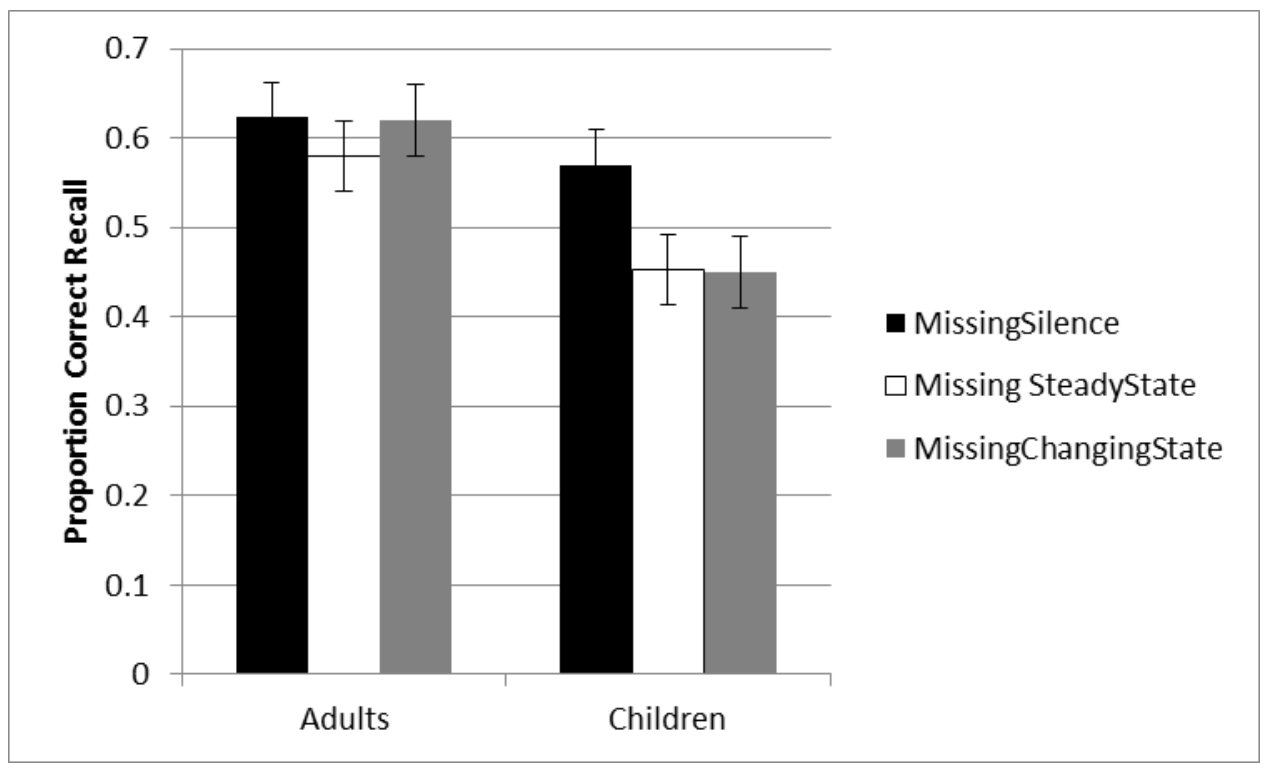

Figure 3. Recall performance in the missing-item task by auditory condition and age-group in Experiment 2. Error bars represent standard error of the mean.

As with the probed-order task data, an arcsine transformation made no difference to the pattern of results. Finally, the data were analyzed without the 17 children who obtained a digit span of 3 . The main effect of auditory condition and the interaction of auditory condition by agegroup remained significant; however, the main effect of age-group was no longer significant, $F(1,109)=1.98, M S E=.14, \eta_{p}{ }^{2}=.02, p=.16$, with the children's mean level of performance now at $M=.55$.

\section{Discussion}

The pattern of results produced in Experiment 2 supports the assumption that children as well as adults are susceptible to interference-by-process, as indicated by the observation of a changing-state effect in the probed-order recall task but not the missing-item task regardless of age-group. However, the results also provide strong support for the view that children's greater susceptibility is due to a deficit in general attentional control abilities (Cowan et al., 2010; Meinhardt-Injac et al., 2015), not a greater susceptibility to interference-by-process: In a task 
generally assumed to be devoid of a requirement for serial rehearsal (the missing-item task), the children showed a relatively large detriment from sound and did so regardless of the nature of that sound (steady or changing). Thus, the results of Experiment 2 suggest that the greater disruption with increasing rehearsal-load for children (Experiment 1) was due to increased rehearsal acting as an increase in general cognitive load. In other words, we argue that, to the extent that children's rehearsal is underdeveloped, this makes them more vulnerable to distraction not directly via an increase in interference-by-process within the specific context of serial short-term memory (e.g., Jones et al., 1992) but indirectly by making it more difficult to maintain attentional control (on any task; e.g., Engle, 2002; Lavie, 2010). As noted, one unexpected feature of the results was a drop in performance for adults in the steady-state, but not changing-state, condition in the missing-item task. While it may be worth following up on this observation in future work, the failure to observe it in previous research using the missing-item task (Jones \& Macken, 1993) leads us not to attempt to ascribe any functional significance to it at present.

\section{General Discussion}

The present findings have implications for theoretical accounts of auditory distraction in verbal short-term memory and highlight the importance of examining cognitive performance at different stages of the lifespan. We demonstrated that both children and adults are negatively affected by irrelevant sound during short-term memory processing but that such susceptibility to distraction differs in important ways at different stages of cognitive development. To summarize, the first experiment manipulated the point at which irrelevant sound was presented to examine the relationship between engagement in rehearsal and susceptibility to distraction. The young adults' data replicated prior research (Macken et al., 1999) as they were most negatively affected 
by irrelevant sound at the end of the presentation phase and during the beginning of the retention interval, points at which the demands on rehearsal would be relatively great. The fact that a similar pattern was found here in children but in exaggerated form is in line with the idea that underdeveloped rehearsal skill plays a role in children's increased sensitivity to irrelevant sound during serial recall. However, based on the duplex-mechanism account of distraction (e.g. Hughes, 2014; Hughes et al., 2007), we reasoned that there are two ways in which underdeveloped rehearsal might account for the particularly acute increase in disruption found across sound-segments for the children: by promoting interference-by-process (e.g., Jones \& Tremblay, 2000) or by imposing a general attentional load that impedes the ability to focus on the task-relevant material. Experiment 2, therefore, examined whether a greater propensity for attentional diversion plays a role in children's particular sensitivity to auditory distraction: Confirming this hypothesis, we found that while there was clear evidence of interference-byprocess in both children and adults — in the form of a changing-state effect in a task involving serial ordering but not in a task devoid of such ordering — there was also evidence that children are particularly prone to attentional diversion as evident from the disruption produced by sound generally (steady or changing) and regardless of the serial-order demands of the focal task. Thus, while children as well as adults are prone to interference-by-process, we suggest that the increased disruptibility in children is due to poorer general attentional control (see also Meinhardt-Injac et al., 2015; Wetzel, 2015). Underdeveloped rehearsal exacerbates this difficulty but does not appear to increase distraction in serial short-term memory directly via increased interference-by-process.

One important implication of the present findings is that they suggest that, at least for some populations, any irrelevant auditory material can potentially draw attention from a focal 
task. While an unexpected deviation in the sound (e.g., Hughes et al., 2007) or a particularly meaningful sound (e.g., one's own name; Röer et al., 2013) is typically necessary to divert most adults' attention from a focal task, if general attentional control ability is very low (such as in children of the age studied here), then such 'extreme' events are unnecessary for attentionaldiversion: Such individuals appear to be prone to having their attention drawn from a focal task even by a repetitive sound. This means that it may sometimes be difficult to identify the manner - interference-by-process or attentional diversion — in which a given changing-state sound-sequence disrupts performance. The present Experiment 2 can be argued to provide an important methodological device for making this identification however: If a given soundsequence is disruptive of serial short-term memory due at least in part to its power to divert attention, then that proportion of the disruption should be isolable by observing its effect, if any, on a task devoid of serial rehearsal (like the missing-item task used here). It is also worth noting that the findings of Experiment 1 indicate that even when irrelevant sound is presented for only 2 $\mathrm{s}$ (in children) or $4 \mathrm{~s}$ (adults) per trial, it is still markedly detrimental to serial recall performance, underscoring the sheer sensitivity of cognitive processing to the mere presence of even fleeting sequences of sound. Furthermore, the view that children's performance was particularly impaired during the retention interval of Experiment 1 partly via attentional diversion is in line with the supposition that such diversion can impair retention processes (see also Röer, Bell, \& Buchner, 2014) and not just stimulus-encoding processes as initially thought (Hughes et al., 2005).

The present findings may also help to reconcile some apparent inconsistencies in the literature on developmental changes in auditory distraction. For example, Klatte et al. (2010) did not find a differential effect of irrelevant sound on the performance of children and adults, but careful scrutiny in light of the current conceptual analysis suggests that the methodology they 
employed may have placed less demand on attentional control. The different irrelevant sound conditions were presented in blocked fashion, as opposed to randomly intermixed as in most irrelevant sound experiments, including the current ones. As noted by the authors themselves, it is plausible that the attention-diverting effect of sound in children was reduced because blocked presentation makes the nature of the upcoming sounds more predictable (cf. Hughes et al., 2013; Vachon, Hughes, \& Jones, 2012). Additionally, the to-be-remembered items were pictures; at recall, all the pictures were re-presented and a line had to be drawn between them in the correct order. Thus, the task demands departed considerably from the verbal recall studies that have found a developmental difference (present study; Elliott, 2002; Elliott et al., 2007; Elliott \& Briganti, 2012).

The present results - particularly those from Experiment 2-also provide a further novel line of evidence for the key claim of the duplex-mechanism account (e.g., Hughes et al., 2007, 2013) that the changing-state effect in serial short-term memory tasks is not due to attentional diversion, contrary to several unitary accounts of auditory distraction (Cowan, 1995; Chein \& Fiez, 2010; Bell, Dentale, Buchner, \& Mayr, 2010; Röer, Bell, \& Buchner, 2015; Röer, Bell, Dentale, \& Buchner, 2011). The unitary view supposes that rather than interfering specifically with serial rehearsal, changing-state stimuli disrupt performance because each change causes an involuntary orienting response (cf. Sokolov, 1963), diverting attention from the focal task whereas with a single steady-state item, the orienting response quickly habituates thereby leaving performance relatively unscathed. There are now several strands of evidence suggesting that the changing-state effect cannot be explained by attentional diversion (for a recent review, see Hughes, 2014). For example, as demonstrated in the present Experiment 2, a changing-state effect is not observed when the focal task is devoid of serial rehearsal (Hughes et al., 2007; Jones 
\& Macken, 1993). It is not clear why this would be the case if changing-state compared to steady-steady stimuli are disruptive because they divert attention from a focal task. Indeed, disruption from an unexpected deviant sound — which all theories agree is caused by attentional diversion - is found regardless of whether the task involves serial rehearsal (Hughes et al., 2007). The present study further undermines the unitary view by showing that in a population (children) for which there is clear evidence of poor attentional control — as evidenced by a strong general effect of sound regardless of task-type - this group still shows no changing-state effect in the context of a task devoid of serial rehearsal.

\section{Some Potential Challenges}

One potential challenge for the poorer-attentional-control view is that older adults-who also tend to have poorer attentional control than young adults (e.g., Gazzaley, Cooney, Rissman, \& D’Esposito, 2005; Hasher \& Zacks, 1988) do not, unlike children, show a greater susceptibility to irrelevant sound (compared to silence) during serial recall compared to young adults (Beaman, 2005; Rouleau \& Belleville, 1996). A possible explanation for this discrepancy, however, is that older adults also exhibit a deficit in the integration of successive sounds into a stream (Trainor \& Trehub, 1989). To the extent that sequential sound integration is a key underpinning of auditory distraction (see Macken, 2014; Macken, Phelps, \& Jones, 2009), such a deficit would reduce the potential for distraction. Thus, poorer top-down attentional control in older adults may in effect be 'compensated for' by poorer bottom-up sound processing. An avenue for further research, therefore, would be to take measures not only of attentional control but also of auditory sequential processing in studies of auditory distraction across different stages of the lifespan. 
A second potential challenge for our account comes from the recent failure to observe a correlation between children's (as well as adults') working memory capacity and their susceptibility to irrelevant sound (Elliott \& Briganti, 2012). The lack of such a correlation for adults is consistent with previous studies (Beaman, 2004; Elliott \& Cowan, 2005; Sörqvist, 2010) and is in line with the supposition that, in the absence of deviations or otherwise highly salient or meaningful stimuli, adults' susceptibility is underpinned primarily by interference-by-process, not attentional diversion. However, the lack of a negative correlation in children is, at first glance, at odds with our account: If working memory capacity reflects attentional control (cf. Engle, 2002) and, as we have argued here, poor attentional control plays a major role in children's increased sensitivity to auditory distraction by any sort of sound, then such a correlation would be expected. However, as in the current Experiment 1, Elliott and Briganti's (2012) study involved a comparison of irrelevant sound with silence. Thus, from the current duplex-mechanism perspective, some of the distraction suffered by children (but not so much the adults) in that study would have been due to interference-by-process which would not be expected to correlate with working memory capacity thereby potentially reducing the chances of observing a correlation between working memory capacity and their overall distractibility. Such considerations again underscore the importance of including steady-state as well as changingstate and silent conditions to isolate different sources of distraction. Indeed, an eminently testable prediction that flows from the current theoretical account is that the disruption from steady-state sound compared to silence in children (and regardless of whether the task involves serial rehearsal) should indeed correlate negatively with their working memory capacity.

\section{Concluding Remarks}


In summary, we have demonstrated here that both serial rehearsal and general attentional control play a role in attentional selectivity and distractibility in serial short-term memory performance. The role of the latter manifests in those with low attentional control due to their stage of cognitive development in the context of any sort of sound but, as shown in other recent studies, those with relatively good attentional control (adults) are more likely to only exhibit such diversion in the presence of unexpected deviants (Hughes et al., 2013), particularly salient stimuli (Röer et al., 2013), or when there is semantic overlap between irrelevant and relevant events (e.g., Beaman, 2004; Marsh, Sörqvist, Hodgetts, \& Beaman, 2015). While serial rehearsal per se plays a role in both children and adults' disruptibility when a task involves serial rehearsal (cf. interference-by-process), it does not appear to play a role in children's increased sensitivity to distractibility.

It is important to note in closing that not only can the study of the developmental aspects of the kind of distraction effects studied here inform theories of the processes involved in shortterm remembering and attentional selectivity, they may also have implications for understanding the educational impact of noise in real-world settings such as classrooms (Klatte, Meis, Sukowski, \& Schick, 2007; Shield \& Dockrell, 2008). The present research suggests that even brief exposure to irrelevant sound can be detrimental to the cognitive performance of children and adults. Additionally, the combination of emerging rehearsal skills and poorer attentional control in young school-aged children (such as those aged 6-8 years) suggests that they will be susceptible to distraction in a broader range of task contexts than adults and by a broader range of types of sound. For example, Klatte et al. (2010) found that $1^{\text {st }}$ grade children were negatively affected by classroom noise, whereas $2^{\text {nd }}$ and $3^{\text {rd }}$ grade children did not show significant disruption from such noise. Thus, it is vital to gain a better theoretical understanding of these 
effects so that we can create optimal learning environments for children at each level of their education. 


\section{References}

Baddeley, A. (2007). Working memory, thought, and action. New York, NY, US: Oxford University Press.

Beaman, C. (2004). The irrelevant sound phenomenon revisited: What role for working memory capacity? Journal of Experimental Psychology: Learning, Memory, and Cognition, 30, 1106-1118. doi:10.1037/0278-7393.30.5.1106

Beaman, C. P. (2005). Irrelevant sound effects amongst younger and older adults: Objective findings and subjective insights. European Journal of Cognitive Psychology, 17, 241 265.

Beaman, C. P., \& Jones, D. M. (1997). Role of serial order in the irrelevant speech effect: Tests of the changing-state hypothesis. Journal of Experimental Psychology: Learning, Memory, and Cognition, 23, 459-471.

Bell, R., Dentale, S., Buchner, A., \& Mayr, S. (2010). ERP correlates of the irrelevant sound effect. Psychophysiology, 47, 1182-1191.

Buschke, H. (1963). Relative retention in immediate memory determined by the missing scan method. Nature, 200, 1129-1130.

Buschke, H. \& Hinrichs, J. V. (1968). Relative vulnerability of item-information in short-term storage for the missing scan. Journal of Verbal Learning and Verbal Behavior, 7, 10431048.

Chein, J. M., \& Fiez, J. A. (2010). Evaluating models of working memory through the effects of concurrent irrelevant information. Journal of Experimental Psychology: General, 139, $117-137$. 
Colle, H. A., \& Welsh, A. (1976). Acoustic masking in primary memory. Journal of Verbal Learning and Verbal Behavior, 15, 17-31.

Cowan, N. (1995). Attention and memory: An integrated framework. New York: Oxford University Press.

Cowan, N., Elliott, E.M., Saults, J.S., Morey, C.C., Mattox, S., Hismjatullina, A., \& Conway, A.R.A. (2005). On the capacity of attention: Its estimation and its role in working memory and cognitive aptitudes. Cognitive Psychology, 51, 42-100.

Cowan, N., Morey, C.C., AuBuchon, A.M., Zwilling, C.E., \& Gilchrist, A.L. (2010). Sevenyear-olds allocate attention like adults unless working memory is overloaded. Developmental Science, 13, 120-133.

Ellermeier, W. \& Zimmer, K. (1997). Individual differences in susceptibility to the "irrelevant speech effect." Journal of Acoustical Society of America, 102, 2191-2199.

Elliott, E. M. (2002). The irrelevant speech effect and children: Theoretical implications of developmental change. Memory and Cognition, 30, 478-487.

Elliott, E. M., Bhagat, S. P., \& Lynn, S. D. (2007). Can children with (central) auditory processing disorders ignore irrelevant sounds? Research in Developmental Disabilities, $28,506-517$.

Elliott, E. M., \& Briganti, A. M. (2012). Investigating the role of attentional processes in the irrelevant speech effect. Acta Psychologica, 140, 64-74.

Elliott, E. M., \& Cowan, N. (2005). Coherence of the irrelevant-sound effect: Individual profiles of short-term memory and susceptibility to task-irrelevant materials. Memory \& Cognition, 33, 664-675. doi:10.3758/BF03195333

Engle, R. W. (2002). Working memory capacity as executive attention. Current Directions in 
Psychological Science, 11, 19-23. doi:10.1111/1467-8721.00160.

Flavell, J. H., Beach, D. R., \& Chinsky, J. M. (1966). Spontaneous verbal rehearsal in a memory task as a function of age. Child Development, 37, 283-299.

Gazzaley, A., Cooney, J. W., Rissman, J., D’Esposito, M. (2005). Top-down suppression deficit underlies working memory impairment in normal aging. Nature Neuroscience, 8, 12981300.

Guttentag, R. (1984). The mental effort requirement of cumulative rehearsal: A developmental study. Journal of Experimental Child Psychology, 37, 92-106.

Hasher, L., \& Zacks, R. T. (1988). Working memory, comprehension, and aging: A review and a new view. In G. H. Bower (Ed.), The psychology of learning and motivation (Vol. 22, pp. 193-225). New York, NY: Academic Press.

Hughes, R. W. (2014). Auditory distraction: A duplex-mechanism account. PsyCh Journal, 3, $30-41$.

Hughes, R. W., Hurlstone, M. J., Marsh, J. E., Vachon, F., \& Jones, D. M. (2013). Cognitive control of auditory distraction: Impact of task difficulty, foreknowledge, and working memory capacity supports duplex-mechanism account. Journal of Experimental Psychology: Human Perception \& Performance, 39, 539-553. doi: 10.1037/a0029064.

Hughes, R. W., \& Jones, D. M. (2001). The intrusiveness of sound: Laboratory findings and their implications for noise abatement. Noise and Health, 4(13), 51-70.

Hughes, R. W., Marsh, J. E., \& Jones, D. M. (2009). Perceptual-gestural (mis)mapping in serial short-term memory: The impact of talker variability. Journal of Experimental Psychology: Learning, Memory, and Cognition, 35, 1411-1425.

Hughes, R. W., Marsh, J. E., \& Jones, D. M. (2011). Role of serial order in the impact of talker 
variability on short-term memory: Testing a perceptual organization-based account. Memory \& Cognition, 39(8), 1435-1447. doi:10.3758/s13421-011-0116-X

Hughes, R. W., Vachon, F., \& Jones, D. M. (2005). Auditory attentional capture during serial recall: Violations at encoding of an algorithm-based neural model? Journal of Experimental Psychology: Learning, Memory, \& Cognition, 31, 736-749.

Hughes, R. W., Vachon, F., \& Jones, D. M. (2007). Disruption of short-term memory by changing and deviant sounds: Support for a duplex-mechanism account of auditory distraction. Journal of Experimental Psychology: Learning, Memory, \& Cognition, 33, 1050-1061.

Humphreys, M. S., \& Schwartz, R. M. (1971). Within category generalization in a missing scan paradigm. Journal of Verbal Learning and Verbal Behavior, 10, 694-701.

Jarrold, C., \& Citroën, R. (2013). Reevaluating key evidence for the development of rehearsal: Phonological similarity effects in children are subject to proportional scaling artifacts. Developmental Psychology, 49, 837-847. doi:10.1037/a0028771

Jones, D. M., Beaman, C. P., \& Macken, W. J. (1996). The object-oriented episodic record model. In S. Gathercole (Ed.), Models of short-term memory (pp. 209-238). London: Erlbaum.

Jones, D. M., Hughes, R. W., \& Macken, W. J. (2006). Perceptual organization masquerading as phonological storage: Further support for a perceptual-gestural view of short-term memory. Journal of Memory and language, 54(2), 265-281.

Jones, D. M., \& Macken, W. J. (1993). Irrelevant tones produce an irrelevant speech effect: Implications for phonological coding in working memory. Journal of Experimental Psychology: Learning, Memory, and Cognition, 19, 369-381. 
Jones, D. M., Macken, W. J., \& Nicholls, A. P. (2004). The phonological store of working memory: Is it phonological and is it a store? Journal of Experimental Psychology: Learning, Memory, and Cognition, 30, 656-674. doi:10.1037/0278-7393.30.3.656

Jones, D. M., Madden, C., \& Miles, C. (1992). Privileged access by irrelevant speech to shortterm memory: The role of changing state. Quarterly Journal of Experimental Psychology, $44 A, 645-669$.

Jones, D. M., \& Tremblay, S. (2000). Interference in memory by process or content? A reply to Neath (2000). Psychonomic Bulletin \& Review, 7, 550-558. doi:10.3758/BF03214370

Klapp, S. T., Marshburn, E. A., \& Lester, P. T. (1983). Short-term memory does not involve working memory of information processing: The demise of a common assumption. Journal of Experimental Psychology: General, 112, 240-264.

Klatte, M., Lachmann, T., Schlittmeier, S., \& Hellbruck, J. (2010). The irrelevant sound effect in short-term memory: Is there developmental change? European Journal of Cognitive Psychology, 22, 1168-1191.

Klatte, M., Meis, M., Sukowski, H., \& Schick, A. (2007). Effects of irrelevant speech and traffic noise on speech perception and cognitive performance in elementary school children. Noise \& Health, 9, 64- 74.

Lavie, N. (2010). Attention, distraction, and cognitive control under load. Current Directions in Psychological Science, 19(3), 143-148.

Lavie, N., Hirst, A., de Fockert, J. W., \& Viding, E. (2004). Load theory of selective attention and cognitive control. Journal of Experimental Psychology: General, 133, 339-354.

LeCompte, D. C. (1996). Irrelevant speech, serial rehearsal, and temporal distinctiveness: A new 
approach to the irrelevant speech effect. Journal of Experimental Psychology: Learning, Memory, and Cognition, 22, 1154-1165.

Lehmann, M., \& Hasselhorn, M. (2012). Rehearsal dynamics in elementary school children. Journal of Experimental Child Psychology, 111, 552-560.

Lewandowsky, S., \& Oberauer, K. (2015). Rehearsal in serial recall: An unworkable solution to the nonexistent problem of decay. Psychological Review, 122(4), 674-699. doi:10.1037/a0039684

Macken, B. (2014). Auditory distraction and perceptual organization: Streams of unconscious processing. PsyCh Journal, 3, 4-16.

Macken, W. J., \& Jones, D. M. (1995). Functional characteristics of the inner voice and inner ear: Single or double agency? Journal of Experimental Psychology: Learning, Memory, \& Cognition, 21, 436-448.

Macken, W. J., Mosdell, N., \& Jones, D. M. (1999). Exploring the irrelevant sound effect: Temporal distinctiveness or changing state? Journal of Experimental Psychology: Learning, Memory, \& Cognition, 25, 810-814.

Macken, W. J., Phelps, F. G., \& Jones, D. M. (2009). What causes auditory distraction? Psychonomic Bulletin \& Review, 16(1), 139-144. doi:10.3758/PBR.16.1.139

Marsh, J. E., Sörqvist, P., Hodgetts, H. M., \& Beaman, C. P. (2015). Distraction control processes in free recall: Benefits and costs to performance. Journal of Experimental Psychology: Learning, Memory, \& Cognition, 41, 118-133.

Meinhardt-Injac, B., Schlittmeier, S., Klatte, M., Otto, A., Persike, M., \& Imhof, M. (2015). Auditory distraction by meaningless irrelevant speech: A developmental study. Applied Cognitive Psychology, 29(2), 217-225. 
Moray, N. (1959). Attention in dichotic listening: Affective cues and the influence of instructions. The Quarterly Journal of Experimental Psychology, 1156-60. doi:10.1080/17470215908416289

Murdock, B. B. Jr. (1968). Serial order effects in short-term memory. Journal of Experimental Psychology, 76, 1-15. doi:10.1037/h0025694

Näätänen, R. (1990). The role of attention in auditory information processing as revealed by event-related potentials and other brain measures of cognitive function. Behavioral and Brain Sciences, 13, 201-288.

Oberauer, K., \& Lewandowsky, S. (2008). Forgetting in immediate serial recall: Decay, temporal distinctiveness, or interference? Psychological Review, 115(3), 544-576.

Röer, J. P., Bell, R., \& Buchner, A. (2013). Self-relevance increases the irrelevant sound effect: Attentional disruption by one's own name. Journal of Cognitive Psychology, 25, 925931.

Röer, J. P., Bell, R., \& Buchner, A. (2015). Specific foreknowledge reduces auditory distraction by irrelevant speech. Journal of Experimental Psychology: Human Perception and Performance. Online First Publication, January 26, 2015. http://dx.doi.org/10.1037/xhp0000028

Röer, J. P., Bell, R., \& Buchner, A. (2014). What determines auditory distraction? On the roles of local auditory changes and expectation violations. PloS one, 9(1), e84166.

Röer, J. P., Bell, R., Dentale, S., \& Buchner, A. (2011). The role of habituation and attentional orienting in the disruption of short-term memory performance. Memory \& Cognition, 39, 839-850.

Rouleau, N. \& Belleville, S. (1996). Irrelevant speech effect in aging: An assessment of 
inhibitory processes in working memory. Journal of Gerontology: Psychological Sciences, 51B, 356-363.

Salamé, P., \& Baddeley, A. D. (1982). Disruption of short-term memory by unattended speech: Implications for the structure of working memory. Journal of Verbal Learning and Verbal Behavior, 21, 150-164.

Schneider, W., Eschman, A., \& Zuccolotto, A. (2002). E-Prime users guide. Pittsburgh, PA: Psychology Software Tools.

Shield, B. \& Dockrell, J. E. (2008). The effects of environmental and classroom noise on the academic attainments of primary school children. Journal of the Acoustical Society of America, 123, 133-144.

Sokolov, Y. N. (1963). Perception and the Conditioned Reflex. Pergamon Press: Oxford.

Sörqvist, P. (2010). High working memory capacity attenuates the deviation effect but not the changing-state effect : Further support for the duplex-mechanism account of auditory distraction. Memory \& Cognition, 38, 651-658.

Sörqvist, P., Marsh, J. E., \& Nöstl, A. (2013). High working memory capacity does not always attenuate distraction: Bayesian evidence in support of the null hypothesis. Psychonomic Bulletin \& Review. Advance online publication. doi: 10.3758/s13423-013-0419-y

Tam, H., Jarrold, C., Baddeley, A. D., \& Sabatos-DeVito, M. (2010). The development of memory maintenance: Children's use of phonological rehearsal and attentional refreshment in working memory tasks. Journal of Experimental Child Psychology, 107, 306-324. doi:10.1016/j.jecp.2010.05.006

Trainor, L. J., \& Trehub, S. E. (1989). Aging and auditory temporal sequencing: Ordering the 
elements of repeating tone patterns. Perception \& Psychophysics, 45, 417-426. doi:10.3758/BF03210715

Turner, M. L., \& Engle, R. W. (1989). Is working memory capacity task dependent? Journal of Memory and Language, 28, 127-154.

Vachon, F., Hughes, R. W., \& Jones, D. M. (2012). Broken expectations: Violation of expectancies, not novelty, captures auditory attention. Journal of Experimental Psychology: Learning, Memory, \& Cognition, 38, 164-177.

Wetzel, N. (2015). Effects of the short-term learned significance of task-irrelevant sounds on involuntary attention in children and adults. International Journal of Psychophysiology, 98, 17-26. doi:10.1016/j.ijpsycho.2015.06.003

Wetzel, N. \& Schröger, E. (2007). Modulation of involuntary attention by the duration of novel and pitch deviant sounds in children and adolescents. Biological Psychology, 75, 24-31. doi:10.1016/j.biopsycho.2006.10.006 